\title{
Außergewöhnliche Manifestation eines Magenkarzinoms mit Meningeosis carcinomatosa und spinaler Metastase
}

\author{
Nicola Cresto ${ }^{1}$, Alain Barth ${ }^{2}$, Marlene Arnold ${ }^{3}$, Rosemarie Weimann ${ }^{3}$, Jürgen Gschossmann ${ }^{4}$, Adrian F. Ochsenbein ${ }^{5}$,
} Christos Kolotas ${ }^{6}$, Hans-Jakob Peter ${ }^{1}$, Uwe Schiemann ${ }^{1}$

\section{ZUSAMMENFASSUNG}

$\square$ Hintergrund: Eine Meningeosis carcinomatosa ist eine seltene Komplikation solider Tumoren, wie Mamma-, Bronchial- oder gastrointestinale Karzinome. Klinisch manifestiert sie sich mit einer äußerst variablen Symptomatik wie z.B. radikulären Schmerzen mit oder ohne neurologische Ausfalle sowie Kopfschmerzen bis hin zu halluzinatorischen Reizsymptomen.

$\square$ Fallbeschreibung: Berichtet wird über den seltenen Fall eines 57-jährigen Patienten mit neurologischen Reiz- und Ausfallsymptomen, hervorgerufen durch eine Meningeosis carcinomatosa und eine spinale Metastase bei einem asymptomatischen Siegelringzellkarzinom des Magens. Trotz kombinierter Radiochemotherapie verstarb der Patient bereits 4 Wochen nach Entlassung aus dem Spital an einer Hirnblutung.

$\square$ Schlussfolgerung: Die Prognose der Meningeosis carcinomatosa ist bis heute unabhängig vom Primärtumor mit einer mittleren Überlebenszeit von 3-4 Monaten sehr schlecht.

Schlüsselwörter: Magenkarzinom $\cdot$ Meningeosis carcinomatosa $\cdot$ Spinale Metastase

Med Klin 2007;102:255-8. DOI 10.1007/s00063-007-1030-7

\section{ABSTRACT}

Extraordinary Manifestation of a Gastric Carcinoma by Leptomeningeal Carcinomatosis and Spinal Metastasis

$\square$ Background: Leptomeningeal carcinomatosis is a rare complication of solid tumors, e.g., breast, lung and gastrointestinal carcinomas. Clinical manifestations are variable with radicular pains with or without neurologic deficiencies as well as headache and hallucinations.

Case Report: The rare case of a 57-year-old patient with neurologic symptoms caused by a leptomeningeal carcinomatosis and a spinal metastasis of an asymptomatic signet-ring cell gastric carcinoma is reported. In spite of combined radiochemotherapy the patient died already 4 weeks after discharge from hospital due to an intracerebral hemorrhage.

\footnotetext{
${ }^{1}$ Klinik für Allgemeine Innere Medizin, Inselspital, Universitätsspital Bern, Schweiz,

${ }^{2}$ Neurochirurgische Klinik, Inselspital, Universitätsspital Bern, Schweiz,

${ }^{3}$ Institut für Pathologie, Inselspital, Universitätsspital Bern, Schweiz,

${ }^{4}$ Gastroenterologische Klinik, Inselspital, Universitätsspital Bern, Schweiz,

${ }^{5}$ Klinik für Onkologie, Inselspital, Universitätsspital Bern, Schweiz,

${ }^{6}$ Klinik für Radio-Onkologie, Inselspital, Universitätsspital Bern, Schweiz.

\section{Fallbeschreibung}

$\square$ Anamnese und klinischer Untersuchungsbefund: Bei dem hier berichteten 57-jährigen Patienten traten seit 3 Monaten intermittierende nächtliche Нypästhesien und Parästhesien im Bereich der lateralen Fußkanten beidseits und im Bereich beider Hände auf. Zudem klagte er über intermittierende Sehstörungen mit Visusabnahme und über eine Schwäche des rechten Beins seit ungefähr 4 Wochen. Anamnestisch ließen sich keine weiteren Begleitsymptome eruieren, insbesondere eine Gewichtsabnahme und gastrointestinale Symptome wurden vom Patienten ausdrücklich verneint. Aus der Vorgeschichte waren eine beidseitige Koxarthrose mit Status nach Hüfttotalprothese rechts und ein Status nach Kataraktoperation beidseits bekannt.

Vom Hausarzt wurden vor der Hospitalisation bereits eine ophthalmologische und eine neurologische Abklärung organisiert. Ophthalmologisch bestanden ein korrigierter Fernvisus von 0,5, eine Quadrantenhemianopsie nasal unten und Stauungspapillen beidseits. Neurologisch konnten eine Pallhypästhesie und Areflexie beidseits festgestellt werden. Es wurden eine vaskuläre Ursache für die Symptomatik angenommen und eine Thrombozytenaggregationshemmung mit 100 mg Acetylsalicylsäure pro Tag verschrieben.

Bei ausbleibender Besserung der Symptomatik wurde der Patient nunmehr zur weitergehenden Abklärung hospitalisiert. Abgesehen von der vorbeschriebenen Quandrantenhemianopsie und einer M4-Parese des Zehenhebers und -beugers links war der übrige klinische Untersuchungsbefund altersentsprechend regelrecht. Im Aufnahmelabor zeigte sich lediglich eine Hypercholesterinämie von $8,0 \mathrm{mmol} / \mathrm{l}$. Die übrigen Routineparameter lagen im Normbereich.

$\square$ Diagnostik: Eine Magnetresonanztomographie der Brust- und Lendenwir- 
belsäule (Abbildung 1) zeigte eine intradurale, kräftig Kontrastmittel aufnehmende Raumforderung in Höhe LWK4-SWK1 mit konzentrischer Kompression des Filum terminale und ein durales Kontrastmittelenhancement. Die Untersuchung des Liquors ergab eine deutliche Pleozytose bei traumatischer Punktion (256 Zellen/ml Liquor, davon 210 Zellen/ml Erythrozyten) und eine Erhöhung des Proteins mit 24 g/l. Die zytologische Beurteilung war auf das Vorliegen einer Meningeosis carcinomatosa verdächtig.

Eine Magnetresonanztomographie des Schädels war bis auf eine postzentrale Einziehung und eine durale Verdickung (vermutet wurde eine alte durale Narbe) unauffällig. Eine Computertomographie des Thorax, des Abdomens und des Beckens zur Tumorsuche zeigte keine Raumforderung sowie keine vergrößerten Lymphknoten. Mittels einer Skelettszintigraphie konnte eine Skelettmetastasierung ausgeschlossen werden.

Zur definitiven Klärung des Befunds wurde eine Hemilaminektomie L4-S1 links mit Exploration und Biopsie des intraduralen Tumors durchgeführt. Intraoperativ war ein derbes tumoröses Gewebe zu erkennen, das die lumbosa-

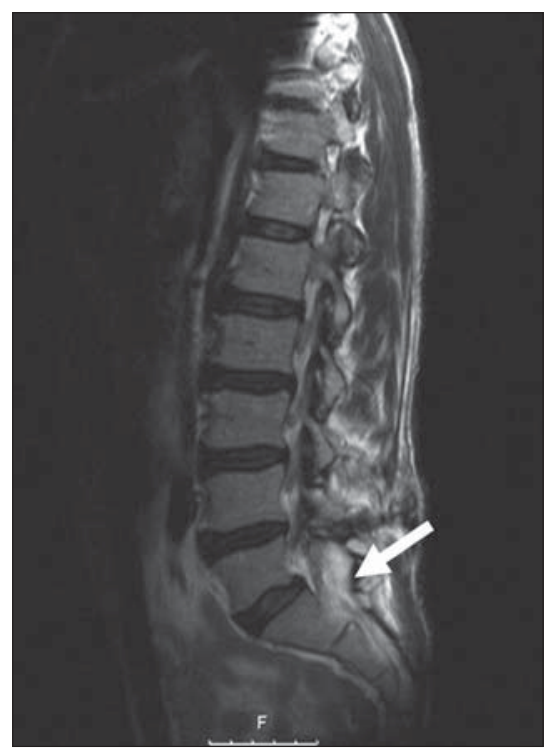

Abbildung 1. Magnetresonanztomographie der BWS und LWS: intradurale, kräftig Kontrastmittel aufnehmende Raumforderung mit konzentrischer Kompression des Filum terminale und duralem Kontrastmittelenhancement.

Conclusion: Until today, prognosis of leptomeningeal carcinomatosis is poor with a median survival between 3-4 months independently of the primary tumor.

Key Words: Gastric carcinoma · Leptomeningeal carcinomatosis · Spinal metastasis

Med Klin 2007;102:255-8 DOI 10.1007/s00063-007-1030-7

krale Wurzel komplett überdeckte. In der Histologie stellte sich ein teils wenig differenziertes, dissoziiert gewachsenes, siegelringzelliges Adenokarzinom dar (Abbildung 2).

Die daraufhin durchgeführte Ösophagogastroduodenoskopie zeigte makroskopisch ein großes, polypoides, derbes, kontaktvulnerables Areal an der Korpushinterwand (Abbildung 3). In der histologischen Untersuchung stellte sich ein exulzeriertes, teils undifferenziertes, teils siegelringzelliges Adenokarzinom (diffuser Typ nach Laurén, G3) dar (Abbildung 4). Endosonographisch hatte der Tumor eine Dicke von $2 \mathrm{~cm}$ und infiltrierte die Serosa. Paragastral waren mehrere, bis $1 \mathrm{~cm}$ große Lymphknoten nachweisbar. $\square$ Diagnose: Aufgrund dieser beiden histologischen Befunde konnte die Diagnose eines metastasierenden Magenkarzinoms mit einer spinalen intraduralen Metastase im Bereich LWK4-SWK1 und einer Meningeosis carcinomatosa gestellt werden. In der TNM-Klassifikation entsprach der Tumor bei Diagnosestellung einem Stadium cT3 cN1 pM1.

\section{DISKUSSION}

Unter Meningeosis carcinomatosa oder karzinomatöser Meningitis versteht man eine Absiedelung von Tumorzellen überwiegend außerhalb des Zentralnervensystems (ZNS) gelegener Primärtumoren in den Liquor. Sie können dann disseminieren und die Leptomeningen an

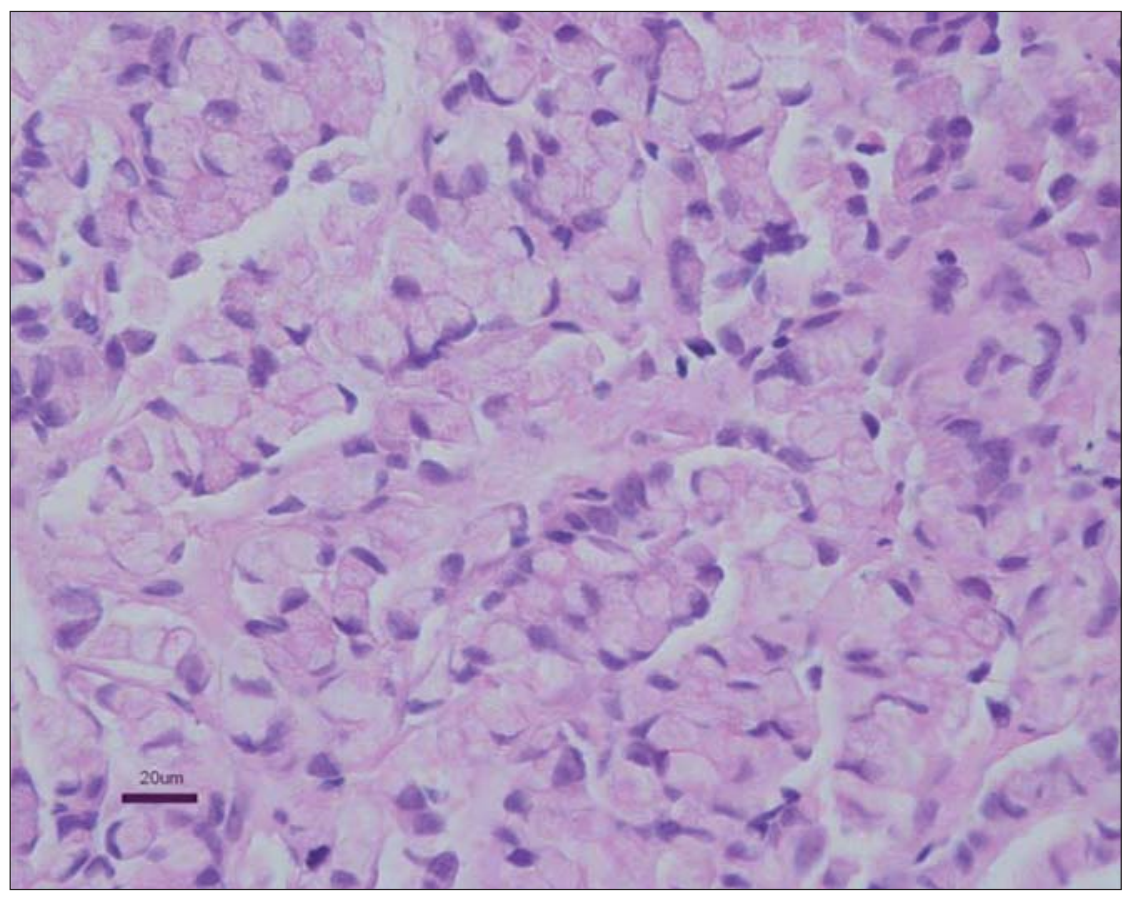

Abbildung 2. Histologie der intraduralen Metastase: wenig differenziertes, dissoziiert gewachsenes, siegelringzelliges Adenokarzinom. 


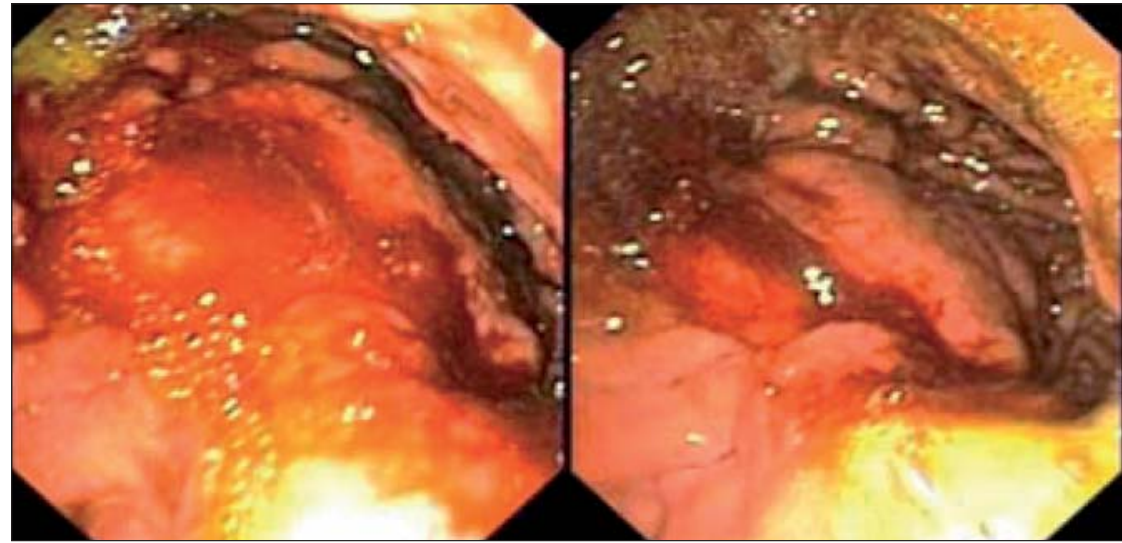

Abbildung 3. Endoskopie des Magens: großes, polypoides, derbes, kontaktvulnerables Areal an der Korpushinterwand.

verschiedenen Stellen befallen. Am häufigsten sind die Hirnbasis, der Sulcus lateralis cerebri (Sylvius-Furche) und die Cauda equina betroffen. Die Tumorzellen können über verschiedene Ausbreitungswege in den Liquor eindringen. Beschrieben ist eine hämatogene Metastasierung in die Ventrikel oder in den Subarachnoidalraum durch arterielle Versorgung oder aus retrogradem Fluss im Batson-Plexus. Die Ausbreitung über perineurale und perivaskuläre Räume oder der Befall benachbarter Strukturen per continuitatem durch direkte Tumorinfiltration spielt ebenfalls eine wichtige Rolle [1]. Eine karzinomatöse Meningi-

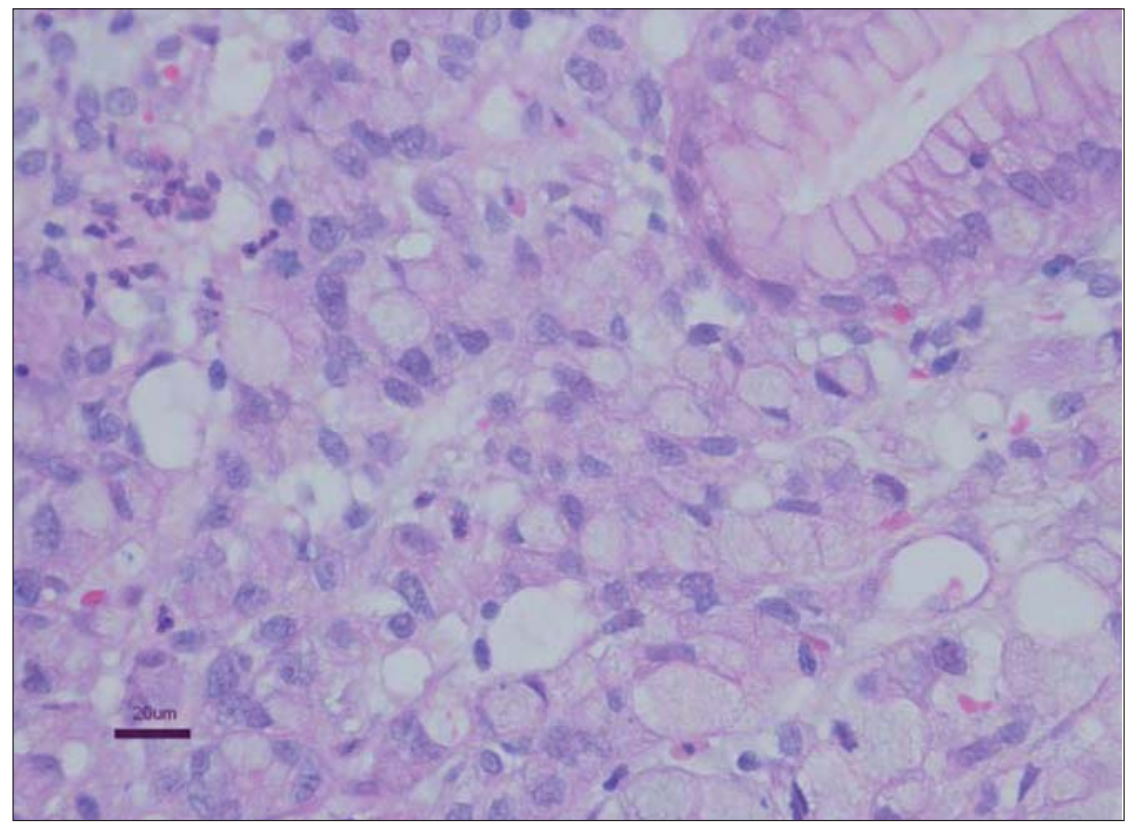

Abbildung 4. Histologie der Magenbiopsie: exulzeriertes, teils undifferenziertes, teils siegelringzelliges Adenokazinom (diffuser Typ nach Laurén, G3).
Aufgrund des multifokalen Geschehens sind die entsprechenden neurologischen Symptome und Ausfälle äußerst variabel. In der Pathogenese der Entstehung der klinischen Symptomatik spielen Kompressionserscheinungen, die einen Hydrozephalus verursachen können, eine direkte Tumorinvasion in Hirn- und Spinalnerven mit entsprechenden Ausfällen sowie eine Zerstörung der Blut-Hirn-Schranke mit Ausbildung eines Hirnödems eine wichtige Rolle. Die Leitsymptome sind am häufigsten radikuläre Schmerzen, Kopfschmerzen und Schmerzen im Bereich der Hals- und der Lendenwirbelsäule [5].

Zur Diagnostik gehören neben einer sorgfältigen Anamnese und der Erhebung des körperlichen Untersuchungsbefunds eine gadoliniumverstärkte Magnetresonanztomographie des Schädels und der Wirbelsäule sowie eine Liquorpunktion. Typische Befunde sind ein diffuses leptomeningeales Kontrastmittelenhancement, intrazerebrale Tumormassen mit evtl. begleitendem Hydrozephalus, multiple noduläre Ablagerungen im Subarachnoidalraum, in den Folia cerebelli oder auf der kortikalen Oberfläche. Die Magnetresonanztomographie der Wirbelsäule zeigt meist eine lineare Kontrastmittelanreicherung im Bereich der ganzen Wirbelsäule oder ein noduläres Enhancement im Bereich der Cauda equina [6, 7]. Ein Kontrastmittelenhancement der Leptomeningen kommt jedoch auch bei Infektionen des ZNS und bei Autoimmunerkrankungen vor oder kann iatrogen durch Zustand nach Radiotherapie oder nach Lumbalpunktion bedingt sein. Aus diesem Grund ist die Liquoruntersuchung zur Sicherung der Diagnose unvermeidbar $[8,9]$.

Beim Vorliegen einer Meningeosis carcinomatosa zeigt die Liquoruntersuchung typischerweise eine lymphozytäre Pleozytose, hohe Proteinkonzentrationen und eine positive Zytologie furr maligne Zellen. Die Spezifität der Liquorzytologie ist sehr hoch, falsch positive Resultate sind äußert selten. Die Sensitivität liegt dagegen nur bei ca. 80-95\% [9].

Die Therapie einer Meningeosis carcinomatosa bei soliden Tumoren ist immer palliativ. Ziele der Behandlung sind eine Linderung der Symptomatik, eine Stabilisierung oder Verbesserung der neurologischen Funktionen und eine Verlängerung der Überlebenszeit. 
Die uns zur Verfügung stehenden Behandlungsmethoden beinhalten eine lokale Radiotherapie (üblicherweise $10 \times 3 \mathrm{~Gy}$ ), eine intrathekale und/oder systemische Chemotherapie oder eine rein supportive Therapie z.B. mit Steroiden. Für keine dieser Therapien ist in randomisierten Studien eine Überlegenheit gegenüber der rein supportiven Therapie nachgewiesen. In der Klinik hat sich eine Therapieentscheidung gemäß dem Risikoprofil und der Symptomatik der Patienten bewährt. Prognostisch günstiger sind chemosensitive Tumoren (z.B. Mammakarzinom), guter Performance-Status, geringe Tumorausbreitung, und außerhalb des ZNS kontrollierte Tumormanifestationen. Diese Patienten profitieren eher von einer Chemotherapie. Für eine intrathekale Therapie stehen Methotrexat, Cytarabin oder Thiotepa zur Verfügung [10-15]. Seit kurzem wird für Cytarabin eine liposomale Form angeboten (DepoCyte), welche nur noch alle 2 Wochen intrathekal verabreicht werden muss [16]. In einer randomisierten Studie konnte eine signifikante Verlängerung der progressionsfreien Überlebenszeit für die Therapie mit liposomalem Cytarabin intrathekal (58 Tage) gegenüber der Standardtherapie mit Methotrexat intrathekal (30 Tage) dokumentiert werden [14]. Dieser relativ geringe Vorteil muss gegen die hohen Kosten der liposomalen Formulierung abgewogen werden. Die Ansprechrate, definiert als das Fehlen maligner Zellen nach intrathekaler Chemotherapie, ist mit 20-60\% relativ hoch. Eine klinische Verbesserung der Symptomatik wird jedoch nur bei 5-15\% der Patienten beschreiben. Allen intrathekalen Therapien gemeinsam ist die Problematik der schwierigen Applikation (bis $2 \times /$ Woche) und der unkontrollierbaren Verteilung im Liquor. Systemische Chemotherapien insbesondere mit hochdosiertem Methotrexat umgehen das Problem der Medikamentenverteilung, sind jedoch wahrscheinlich der intrathekalen Therapie nicht überlegen.

Bei Patienten mit schlechtem Risikoprofil ist die Prognose trotz chemotherapeutischer Behandlung infaust. In diesen Fällen steht vor allem eine supportive Therapie mit Kortikosteroiden und erforderlichenfalls mit Antiepileptika im Vordergrund.

Eine wichtige Rolle in der Therapie der Meningeosis carcinomatosa spielt die zusätzliche palliative perkutane Radiotherapie (30-36 Gy mit Einzeldosen von 3 Gy) oder eine chirurgische Entlastung in den Fällen, in denen eine Kompressionssymptomatik im Vordergrund steht $[5,17,18]$. Die perkutane Radiotherapie bringt häufig schneller eine Symptomverbesserung als die Chemotherapie, insbesondere bei Massenläsionen (Bulk) oder bei Behinderung des Liquorabflusses.

Trotz allem ist die Prognose einer Meningeosis carcinomatosa sehr schlecht. In vier kontrollierten, randomisierten Studien betrug das mittlere Überleben trotz Chemotherapie nur 2-4 Monate ab Diagnosestellung [12].

Wir entschlossen uns nach Hemilaminektomie im Bereich L4-S1 zu einer postoperativen Radiotherapie in Kombination mit einer systemischen Chemotherapie mit 5-Fluorouracil und Cisplatin. Ein begleitendes Hirnödem (klinisch waren u.a. Halluzinationen aufgetreten) wurde mit hochdosierten Steroidgaben (Dexamethason bis $24 \mathrm{mg}$ p.o. täglich) therapiert. Trotz dieser Maßnahmen verstarb der Patient leider bereits 4 Wochen nach Entlassung aus dem Spital an einer akuten Hirnblutung.

Der vorliegende Fall ist insbesondere deshalb außergewöhnlich, weil die Meningeosis carcinomatosa zur Diagnose führte und nicht erst im Verlauf der Erkrankung auftrat. Wenige ähnliche Fälle sind in der Literatur beschrieben [19-24]. Bei unspezifischen neurologischen Symptomen wie im vorliegenden Fall sollte man differentialdiagnostisch eine Meningeosis carcinomatosa unbedingt in $\mathrm{Be}-$ tracht ziehen.

\section{Literatur}

1. Kokkoris CP. Leptomeningeal carcinomatosis. How does cancer reach the pia-arachnoid? Cancer 1983; 51:154-60.

2. Kaplan JG, DeSouza TG, Farkash A, et al. Leptomeningeal metastases: comparison of clinical features and laboratory data of solid tumors, lymphomas and leukemias. J Neurooncol 1990;9:225-9.

3. Posner JB. Neurologic complications of cancer. Philadelphia: Davis, 1995

4. Lisenko Y, Kumar AJ, Yao J, et al. Leptomeningeal carcinomatosis originating from gastric cancer: report of eight cases and review of the literature. Am J Clin Oncol 2003;26:165-70

5. Wasserstrom W, Glass JP, Posner JB. Diagnosis and treatment of leptomeningeal metastases from solid tumors: experience with 90 patients. Cancer 1982;49:759-72

6. Chamberlain MC, Sandy AD, Press GA. Leptomeningeal metastasis: a comparison of gadolinium-enhanced MR and contrast-enhanced CT of the brain. Neurology 1990;40:435-8.

7. Chamberlain MC. Comparative spine imaging in leptomeningeal metastases. J Neurooncol 1995;23: 233-8.
8. Schumacher M, Orszagh M. Imaging techniques in neoplastic meningeosis. J Neurooncol 1998;38: $111-20$.

9. Freilich RJ, Krol G, DeAngelis LM. Neuroimaging and cerebrospinal fluid cytology in the diagnosis of leptomeningeal metastasis. Ann Neurol 1995;38: 51-7.

10. Siegal T, Lossos A, Pfeffer MR. Leptomeningeal metastases: analysis of 31 patients with sustained off-therapy response following combined-modality therapy. Neurology 1994;44:1463-9.

11. Grossman SA, Finkelstein DM, Ruckdeschel JC, et al. Randomized prospective comparison of intraventricular methotrexate and thiotepa in patients with previously untreated neoplastic meningitis. Eastern Cooperative Oncology Group. J Clin Oncol 1993;11: 561-9.

12. Glantz MJ, Cole BF, Recht L, et al. High-dose intravenous methotrexate for patients with nonleukemic leptomeningeal cancer: is intrathecal chemotherapy necessary? J Clin Oncol 1998;16:1561-7.

13. Glantz MJ, Jaeckle KA, Chamberlain MC, et al. A randomized controlled trial comparing intrathecal sustained-release cytarabine (DepoCyt) to intrathecal methotrexate in patients with neoplastic meningitis from solid tumors. Clin Cancer Res 1999;5:3 394-402.

14. Cole BF, Glantz MJ, Jaeckle KA, et al. Quality-of-life-adjusted survival comparison of sustained-release cytosine arabinoside versus intrathecal methotrexate for treatment of solid tumor neoplastic meningitis. Cancer 2003;97:3053-60.

15. Hitchins RN, Bell DR, Woods RL, et al. A prospective randomized trial of single-agent versus combination chemotherapy in meningeal carcinomatosis. J Clin Oncol 1987;5:1655-62.

16. Rueda DA, Olmos HD, Viciana G, et al. Liposomal cytarabine (DepoCyte) for the treatment of neoplastic meningitis. Clin Transl Oncol 2005;7:232-8.

17. Herrmann B, Hultenschmidt B, Sautter-Bihl ML. Radiotherapy of the neuroaxis for palliative treatment of leptomeningeal carcinomatosis. Strahlenther Onkol 2001;177:195-9.

18. Omuro AM, Lallana EC, Bilsky MH, et al. Ventriculoperitoneal shunt in patients with leptomeningeal metastasis. Neurology 2005;64:1625-7.

19. Agnelli G, Giresele P. Mucus-secreting "signet-ring" cells in CSF revealing the site of primary cancer. Postgrad Med J 1980;56:868-70.

20. McCrary JA 3rd, Patrinely JR, Font RL. Progressive blindness caused by metastatic occult signet-ring cell gastric carcinoma. Arch Ophthalmol 1986;104: 410-3.

21. Grove A. Meningeal carcinomatosis from a clinically undiagnosed early gastric cancer. Pathol Res Pract 1991;187:341-5.

22. Deeb LS, Yamout BI, Shamseddine AI, et al. Meningeal carcinomatosis as the presenting manifestation of gastric adenocarcinoma. Am J Gastroenterol 1997; 97:329-31.

23. Lee JL, Kang YK, Kim TW, et al. Leptomeningeal carcinomatosis in gastric cancer. J Neurooncol 2004; 66:167-74.

24. Braeuninger S, Mawrin C, Malfertheiner P, et al. Gastric adenocarcinoma with leptomeningeal carcinomatosis as the presenting manifestation: an autopsy case report. Eur J Gastroenterol Hepatol 2005;17: 577-9.

\section{Korrespondenzanschrift}

Priv.-Doz. Dr. Uwe Schiemann

Klinik und Poliklinik

für Allgemeine Innere Medizin

Inselspital Bern

Freiburgstraße

3010 Bern

Schweiz

Telefon (+41/31) 632-2111

Fax -4814

E-Mail:uwe.schiemann@insel.ch 